\title{
Peripheral Synapses at Identified Mechanosensory Neurons in Spiders: Three-Dimensional Reconstruction and GABA Immunocytochemistry
}

\author{
Ruth Fabian-Fine,, ${ }^{1,2}$ Ulli Höger, ${ }^{1}$ Ernst-August Seyfarth, ${ }^{1}$ and lan A. Meinertzhagen ${ }^{2}$ \\ 1Zoologisches Institut, J. W. Goethe-Universität, D-60054 Frankfurt am Main, Germany, and 2Neuroscience Institute, \\ Dalhousie University, Halifax, Nova Scotia, Canada B3H 4J1
}

The mechanosensory organs of arachnids receive diverse peripheral inputs. Little is known about the origin, distribution, and function of these chemical synapses, which we examined in lyriform slit sense organ VS-3 of the spider Cupiennius salei. The cuticular slits of this organ are each associated with two large bipolar mechanosensory neurons with different adaptation rates. With intracellular recording, we have now been able to correlate directly the staining intensity of a neuron for acetylcholinesterase with its adaptation rate, thus allowing us simply to stain a neuron to identify its functional type. All rapidly adapting neurons stain more heavily than slowly adapting neurons. Immunostaining of whole-mount preparations reveals GABA-like immunoreactive fibers forming numerous varicosities at the surface of all sensory neurons in VS-3; peripheral GABA-like immunoreactive somata are lacking. Sectioning the leg nerve procures rapid degeneration of most fiber profiles, confirming that the fibers are efferent. Punctate synapsin-like immunoreactivity colocalizes to these varicosities, although some synapsin-like immunoreactive puncta are GABAimmunonegative. Fibers with similar immunoreactivities are also associated with trichobothria, tactile hairs, internal joint receptors, i.e. other types of spider mechanosensory organs. In organ VS-3, immunoreactivity is most dense across the initial axon segment. The exact distribution of peripheral synapses was reconstructed from a $10-\mu \mathrm{m}$-long electron micrograph series of the dendritic, somatic, and initial axon regions of acetylcholinesterase-stained VS-3 neurons. These reveal a pattern similar to that of the synapsin-like immunoreactivity. Two different types of synapse were distinguished on the basis of their presynaptic vesicle populations. Many peripheral synapses thus appear to derive from efferent GABA-like immunoreactive fibers and probably provide centrifugal inhibitory control of primary mechanosensory activities.

Key words: peripheral synapses; mechanoreceptors; synapsin; efferent control; immunocytochemistry; three-dimensional reconstruction; ultrastructure; spider; acetylcholinesterase
Efferent control of peripheral receptor neurons from the CNS is a widespread mechanism in the animal kingdom (Livingston, 1959), but among all arthropod integumentary sensilla, peripheral synaptic structures have been described only at the sensory neurons of chelicerates (Foelix, 1975, 1985). The function of this innervation remains enigmatic, except in two cases. In the first example, for the giant fiber neurons in the antenniform forelegs of whip spiders and whip scorpions, it has been suggested that input originating from mechanosensory bristles may be integrated by the giant neuron before being rapidly conducted to the CNS (Foelix and Troyer, 1980). In that case, however, the peripheral synapses arise predominantly from afferent inputs and not from

Received Aug. 10, 1998; revised Oct. 12, 1998; accepted Oct. 16, 1998.

This work was supported by Deutsche Forschungsgemeinschaft Grant Se367/2-3 (E.-A.S.), Deutscher Akademischer Austauschdienst Grant 315 ( R.F.-F.), and Natural Sciences and Engineering Research Council (Ottawa) Grant OPG0000065 (I.A.M.). The monoclonal antibody against Drosophila synapsin was kindly provided by Erich Buchner (Universität Würzburg). We thank Zhiyuan Lu and Beata Krebs for extensive help with ultramicrotomy and electron microscopy, Istvan Molnar for help with computer reconstructions, Xue J. Sun for help with microscopy, Martin Jatho for help with computer work, and Alan M. Fine for helpful discussions.

Correspondence should be addressed to Dr. Ian A. Meinertzhagen, Neuroscience Institute, Life Sciences Centre, Dalhousie University, Halifax, Nova Scotia, Canada B3H $4 \mathrm{~J} 1$.

Dr. Fabian-Fine's present address: Department of Biology, The Open University, Walton Hall, Milton Keynes MK7 6AA, Bucks, UK.

Dr. Höger's present address: Department of Physiology and Biophysics, Sir Charles Tupper Medical Building, Dalhousie University, Halifax, Nova Scotia, Canada B3H 4H7.

Copyright (ㄷ) 1998 Society for Neuroscience $\quad 0270-6474 / 98 / 190298-13 \$ 05.00 / 0$ efferent innervation. Only the second case, for the eccentric cells in the Limulus compound eye, provides a clear example of the function of peripheral synapses onto a chelicerate sensory neuron (Fahrenbach, 1985) during lateral inhibition (Hartline and Ratliffe, 1972). Here, we seek comparable evidence for the role of peripheral innervation to various mechanoreceptors of spiders.

To understand the function of spider mechanosensory synapses, detailed knowledge of the distribution, neurotransmitter content, fine structure, and presynaptic origin of these contacts is required. We have therefore examined the number and distribution of peripheral synapses on single, identified mechanosensory neurons in the compound slit sense organ VS-3 of the Central American hunting spider Cupiennius salei (Barth and Libera, 1970, their nomenclature). In addition, we provide novel evidence that GABA is a neurotransmitter candidate at such peripheral synapses, not only in slit sense organs but also at the neurons of three other mechanoreceptor types in the spider leg: trichobothria, tactile hairs, and internal joint receptors.

Slit sense organs detect minute mechanical strains in the cuticular exoskeleton of arachnids (Barth, 1985; Zill and Seyfarth, 1996) and have cuticular specializations in the form of slits, each with an associated pair of bipolar mechanosensory neurons (Seyfarth et al., 1985). Tactile hairs are supplied by a set of three bipolar mechanosensory neurons, which are distinguishable by their size and different electrophysiological properties (Harris and Mill, 1977; Eckweiler and Seyfarth, 1988). Trichobothria (filiform hairs) detect air-particle movements (Barth et al., 1995) 


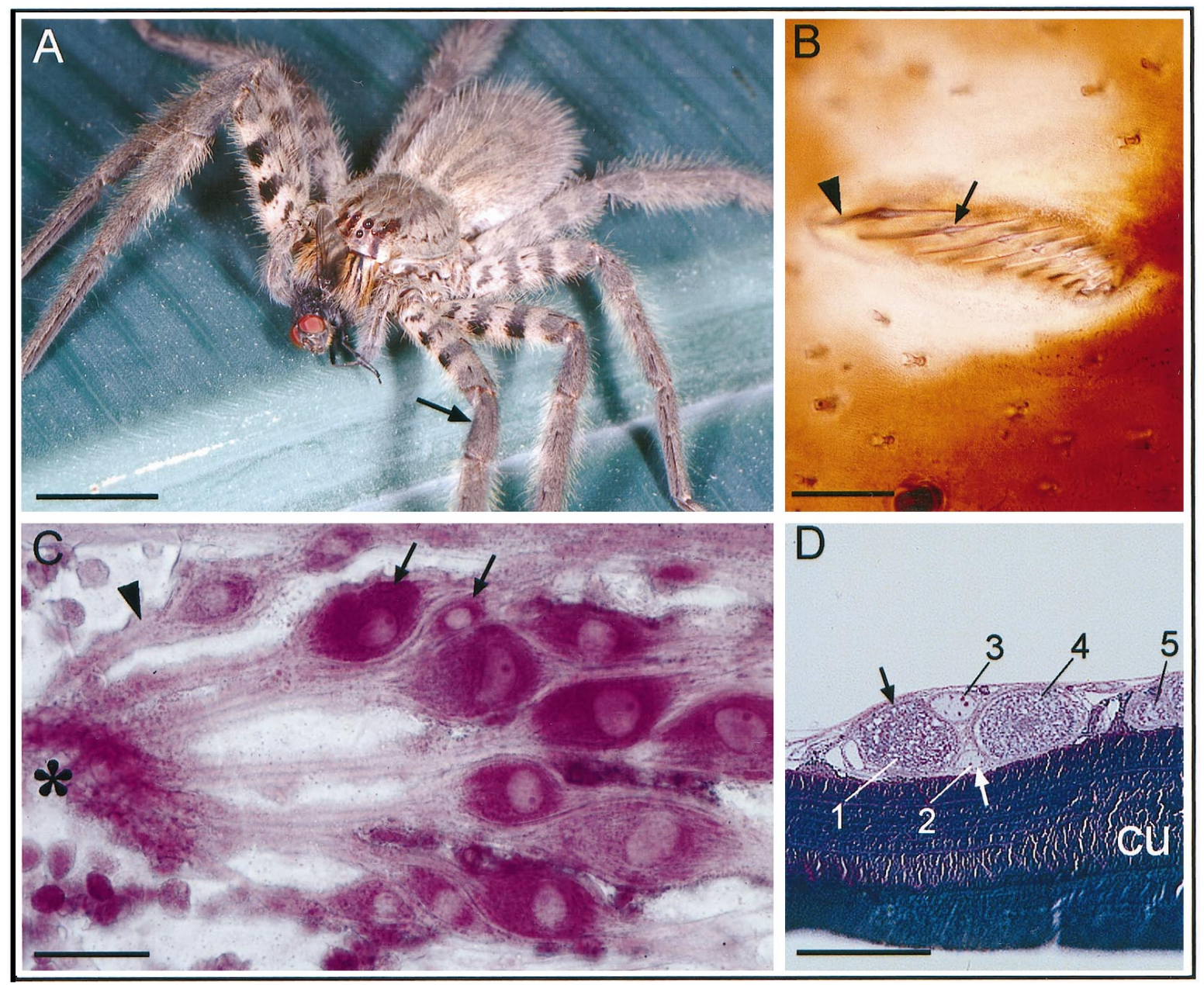

Figure 1. The lyriform slit sense organ VS-3 of the spider's leg. A, Cupiennius salei Keys., adult female. Arrow, Position of the VS-3 organ on the anterior surface of the leg patella. Scale bar, $1 \mathrm{~cm}$. B. External cuticular features of a VS-3 organ with nine cuticular slits seen by light microscopy. Slit 1 (arrowhead) lies both more dorsal and more distal along the patella than slit 9. The clear region at the center of each slit (slit 2 , arrow) represents the site of dendritic insertion. Magnification, $260 \times$. Scale bar, $50 \mu \mathrm{m}$. $C$, Whole-mount (sensory hypodermis) preparation of mechanosensory neurons beneath the cuticle in a VS-3 organ, consisting of seven slits. Cresyl violet stain is the same orientation as in $B$. Each slit is associated with a pair of bipolar neurons that are enveloped by a glial sheath (arrowhead). Asterisk, Dendrite tip region of the neurons. The two neurons belonging to slit 2 (arrows) are clearly distinguishable from each other by their different soma sizes. Magnification, $300 \times$. Scale bar, $50 \mu \mathrm{m} . D$, Neuron pairs in the third $(1,2)$, the fourth $(3,4)$, and one neuron in the fifth (5) slit of a VS-3 organ; $1 \mu \mathrm{m}$ semithin transverse section. The neuron pair associated with the third slit (black arrow, Type a neuron; white arrow, Type b neuron) is reconstructed from serial sections in Figure 5. cu, Cuticle. Magnification, $340 \times$. Scale bar, $50 \mu \mathrm{m}$.

and are located on the distal leg segments; they are associated with three or four bipolar mechanosensory neurons (Reissland and Görner, 1985). The internal joint receptors examined here lie in the femur-patella joint (R9 and R11) (Rathmayer and Koopmann, 1970, their nomenclature; Seyfarth et al., 1985) and comprise 10 mechanosensory neurons (Foelix and Choms, 1979). The cell bodies of all these mechanosensory sensilla are embedded in the hypodermis, the delicate epithelium underlying the cuticle, close to the area of their dendritic insertion in the periphery. These mechanosensors have been implicated in proprioception, in the control of joint movements, in prey localization, and in other orientation behaviors (Seyfarth, 1985; Zill and Seyfarth, 1996). They constitute prominent, accessible identified neurons, with peripheral synaptic inputs, the action of which we now seek to clarify. A preliminary version of this paper has appeared previously (Fabian et al., 1998).

\section{MATERIALS AND METHODS}

Animals. Central American wandering spiders, Cupiennius salei Keys. (Ctenidae), were raised in the laboratory (Höger and Seyfarth, 1995).
Animals were kept individually in glass containers and fed with crickets weekly. Adults older than 10 months were used for all preparations (Fig. $1 A)$. The spiders were anesthetized by exposure to carbon dioxide. All experiments followed established principles of laboratory animal care and complied with the specific respective national laws.

Dissection. The dendrites, somata, and initial axon segments of the mechanosensory neurons we examined are located in the hypodermis, a delicate epithelium underlying the cuticular exoskeleton (Fig. 1D). For the initial steps of all histological procedures applied here, we used isolated parts of the walking legs. Deeply anesthetized spiders were perfused (via the heart) with freshly made fixative (see below). Legs were removed from the prosoma, split longitudinally, and then immersed under fixative for $1-2 \mathrm{hr}$ at $4^{\circ} \mathrm{C}$. Fine dissection of isolated leg parts, with the hypodermis and neuronal structures still attached, was then performed in PBS $(0.1 \mathrm{M}$ phosphate buffer, including $\mathrm{NaCl}$ to a final concentration of $0.08 \mathrm{M}$, adjusted to $\mathrm{pH} 7.3$ ). In the following, we refer to such preparations as "cuticular preparations." Before light-microscopic examination of (immuno-) stained neurons, the hypodermis and associated neuronal structures were cautiously detached from the cuticle and transferred onto gelatin-coated slides as whole-mount preparations (Fig. $1 C)$; these will be referred to as "sensory hypodermis preparations" (Fig. 1C).

Removal of the spider CNS and embedding in agarose followed the 
methods described previously (Seyfarth et al., 1993). Agarose-embedded CNS preparations were subsequently sectioned at $50 \mu \mathrm{m}$ with a Vibratome (Series 1000; Technical Products International, St. Louis, MO). All procedures for tissue fixation and staining of CNS samples were identical with those applied to the cuticular preparations.

Degeneration tests. We used peripheral degeneration to seek evidence for putative efferent fibers contacting mechanoreceptors, by amputating the legs close to the cephalothorax in deeply anesthetized animals. Isolated legs were then transferred to Cupiennius saline (Maier et al., 1987) at $23^{\circ} \mathrm{C}$ for $1-8 \mathrm{hr}$ before treatment with electron microscopy (EM) fixative and processed for EM thereafter (see below). This range of degeneration times was chosen to secure the orthograde degeneration of the efferent fibers without causing retrograde degeneration of the primary mechanosensory neurons.

Acetylcholinesterase histochemistry. Acetylcholinesterase (AChE) histochemical staining was performed according to the "direct coloring" technique of Karnovsky and Roots (1964), modified as described previously (Fabian and Seyfarth, 1997). The method yields a reddish-brown precipitate directly at the site of enzymatic activity. After amputation, legs were left in spider saline (at room temperature for $15 \mathrm{~min}$ ) to replenish synaptic vesicle pools (Wickelgren et al., 1985) and then fixed briefly $(5 \mathrm{~min})$ in $4 \%$ paraformaldehyde in PBS (PFA). After fine dissection, cuticular preparations were further fixed in 4\% PFA for 35 min. Subsequently, tissue was incubated in the staining solution for $9 \mathrm{hr}$ at $4^{\circ} \mathrm{C}$, rinsed in PBS, post-fixed in $3 \%$ glutaraldehyde (GA) in PBS for $15 \mathrm{~min}$, and then processed for EM as described below.

Electron microscopy. Preparations intended for conventional EM were fixed in $3 \% \mathrm{GA}$, rinsed in PBS $(4 \times$ each $10 \mathrm{~min})$, osmicated in $0.5 \%$ $\mathrm{OsO}_{4}-\mathrm{PBS}$ for $15 \mathrm{~min}$, dehydrated in a graded series of ethanol, and then changed into propylene oxide before embedment in Araldite (13824; Serva Feinbiochemica, Heidelberg, Germany). The resin was polymerized in embedding molds at $60^{\circ} \mathrm{C}$, and series of $75 \mathrm{~nm}$ sections, each totaling $10 \mu \mathrm{m}$ depth, were cut with a Reichert Ultracut S. All sections were collected on Pioloform-coated single-slot copper grids and contrasted with aqueous $1.5 \%$ uranyl acetate and Reynolds's lead citrate, before examination in a Philips 201C or a Hitachi H-500 transmission electron microscope. To reconstruct the two neurons associated with the third slit in a VS-3 organ, 1- $\mu$ m-thick serial sections were collected from the areas between those used for EM reconstruction. These were mounted on slides and stained with a fuchsin and methylene blue/azure mixture (Humphrey and Pittman, 1974).

Cresyl violet staining. Cuticular preparations were fixed in $6 \%$ formaldehyde for $1 \mathrm{hr}$, rinsed in acetate buffer ( $0.1 \mathrm{M}, \mathrm{pH} 5.6)$, and left in $1.5 \%$ cresyl violet (5235; Merck, Darmstadt, Germany) in acetate buffer for 3 min. After rinsing, stained hypodermis preparations were mounted and immediately photographed on Eastman Kodak (Rochester, NY) Ektachrome 400 film using an Axiophot microscope (Zeiss, Oberkochen, Germany).

Synapsin immunostaining. Cuticular preparations of all four sensilla types investigated here were fixed in $4 \%$ PFA, washed in PBS $(4 \times$ each $10 \mathrm{~min}$ ), and permeabilized in $0.1 \%$ saponin in PBS for $15 \mathrm{~min}$. They were then incubated with a mouse monoclonal antibody against Drosophila synapsin (SYNORF1) (Klagges et al., 1996) for $12 \mathrm{hr}$ at $4^{\circ} \mathrm{C}$. The antibody was used 1:100 in a dilution and blocking medium (DBM) consisting of $3 \%$ skim milk powder (Carnation, Glendale, CA), $0.25 \%$ bovine serum albumin (BSA) (A-9647; Sigma, Deisenhofen, Germany), $3 \%$ normal goat serum, and $0.005 \%$ saponin (S-2149; Sigma) in PBS. After washing in PBS $(4 \times$ each $10 \mathrm{~min})$, preparations were incubated at $4^{\circ} \mathrm{C}$ overnight with Cy3-coupled goat anti-mouse IgG (Jackson ImmunoResearch, West Grove, PA) diluted 1:600 in DBM. After final rinses in PBS $(5 \times$ each $10 \mathrm{~min})$, sensory hypodermis preparations were mounted in Mowiol (CM-B33500; Hoechst, Frankfurt, Germany). Neurons were examined using a Zeiss Axiophot microscope with epifluorescence optics. Bright red Cy3-immunofluorescent labeling was detected using green excitation (excitation filter, bandpass 546/12; barrier filter, low-pass 520 ) and photographed immediately (color, Ektachrome 400; black and white, Ilford Delta 400; Ilford, Mobberley, UK).

GABA immunostaining. Anesthetized animals were perfused with $0.1 \%$ GA- $1 \%$ picric acid in $4 \%$ PFA, and amputated legs were kept in the same fixative at $4^{\circ} \mathrm{C}$ for $2 \mathrm{hr}$. After rinsing in PBS ( $4 \times$ each $10 \mathrm{~min}$ ), cuticular preparations were incubated for $30 \mathrm{~min}$ in PBS containing $0.1 \%$ sodium borohydride to unmask antigens (Seguela et al., 1984) and $0.1 \%$ glycine to quench residual aldehydes (Mulloney and Hall, 1990). After repeated rinsing in PBS, preparations were exposed to $0.1 \%$ saponin in PBS (15 min) to permeabilize cell membranes. Tissue was then incubated overnight at $4^{\circ} \mathrm{C}$ with rabbit polyclonal antiserum against GABA (AMP 300: Société Française de Recherches et d'Investissements Laboratoire, St. Jean d'Illac, France) diluted 1:4,000 in DBM. After rinsing and renewed incubation in $0.1 \%$ saponin in PBS (15 min), preparations were incubated with Cy3-conjugated goat anti-rabbit IgG (1:450 in DBM; Jackson ImmunoResearch) at $4^{\circ} \mathrm{C}$ for $12 \mathrm{hr}$. Immunofluorescence was examined as described above, with green fluorescein isothiocyanate (FITC) fluorescence detected via blue excitation (excitation filter, bandpass 450-490; barrier filter, low-pass 520).

Double immunolabeling. Double staining with antibodies against both synapsin and GABA was performed to examine the extent of colocalization between the two patterns of immunolabeling in the peripheral innervation. Preparations were processed for GABA immunostaining as described above, except that the secondary antibody used was FITCcoupled goat anti-rabbit IgG (1:150 in DBM; F-9887; Sigma) and the monoclonal antibody against synapsin was added to the primary GABA antibody solution $4 \mathrm{hr}$ after the latter was first applied. Primary and secondary antibodies were used at the same concentrations as mentioned above. Separate and double photographic exposures were taken at the appropriate filter settings.

Controls. Three different types of control preparations were made: (1) immunostaining of CNS preparations with known GABAergic neurons was performed on $50 \mu \mathrm{m}$ tissue sections of agarose-embedded CNS preparations post-fixed in $0.1 \% \mathrm{GA} / 1 \%$ picric acid in $4 \%$ PFA for $12 \mathrm{hr}$ at $4^{\circ} \mathrm{C}$; (2) preparations were immunostained as described above, except that they were incubated in DBM lacking the primary antibody; and (3) preparations were immunostained as described above, except that the primary antibody was preadsorbed for $1 \mathrm{hr}$ before incubation with a GABA-BSA-GA conjugate, made by combining 5 mM GABA (A-2129; Sigma) and $5 \mathrm{mg} / \mathrm{ml} \mathrm{BSA}$ in $1 \% \mathrm{GA}$, as described previously (Walrond et al., 1993).

Electrophysiological typing. To characterize and type the electrical responses of VS-3 neurons according to their adaptation behavior, intracellular recordings were made with glass microelectrodes in isolated cuticular preparations of the organ, similar to methods described by Seyfarth and French (1994). Briefly, microelectrodes were filled with $3 \mathrm{M}$ $\mathrm{KCl}$ and connected to a conventional electrometer amplifier with bridge compensation for electrode resistance during current injection. VS-3 neurons were identified visually, and microelectrodes were lowered onto the somata of selected neurons through a thin layer of Cupiennius saline (Maier et al., 1987) with the aid of a Leitz (Wetzlar, Germany) micromanipulator. Cell penetration was achieved by gentle tapping of the manipulator; electrode resistances were 45-75 $\mathrm{M} \Omega$, with time constants of $1-3 \mu \mathrm{sec}$ in solution. For electrical stimulation via the recording electrode, current steps were produced by a stimulator (SD9; Grass, Quincy, MA) and maintained for at least $200 \mathrm{msec}$. Current and voltage recordings were stored on a digital audio tape recorder (DTR-1802; Biologic, Claix-Grenoble, France) for later analysis. After a neuron was typed as either rapidly adapting (Type a) or slowly adapting (Type b), the preparation was immediately fixed in $4 \%$ PFA for subsequent AChE histochemistry as described above.

Three-dimensional reconstructions. The three-dimensional surfaces of one pair of VS-3 neurons were reconstructed from consecutive thinsection profiles. Consecutive EM negatives were manually aligned, captured into an IBM-compatible computer with frame-grabbing software (AMICUS; ISG Technologies, Mississauga, Ontario, Canada), digitized, and manually segmented using a graphics work station (ISG Technologies) running three-dimensional reconstruction software (ICAR 80.8, version 5.0.1; ISG Technologies) as described previously (Meinertzhagen and Hu, 1996; Meinertzhagen et al., 1998). Light-microscopic images of sequential semithin sections were captured using a Zeiss Axiophot microscope equipped with a video camera and processed as above.

Morphometry and quantitative analysis. We quantified the size and distribution of immunoreactive sites in two ways. First, we used vernier calipers to measure the mean sizes of varicosities from GABA-like immunoreactive (GABA-LIR) fibers from their enlarged negative images projected on a white surface. Second, to estimate the extent of colocalization between synapsin-like immunoreactive (SYN-LIR) and GABA-LIR sites, we compared the number and distribution of synapsinlike immunofluorescent puncta (IR-puncta) and GABA-LIR varicosities in identical areas of double-stained sensory hypodermis preparations, which included the sensory neurons of trichobothria and tactile hairs. Photographic images of each single component (i.e., synapsin and GABA immunofluorescence) were projected separately, and the SYN-LIR puncta or GABA-LIR varicosities were marked and counted over iden- 

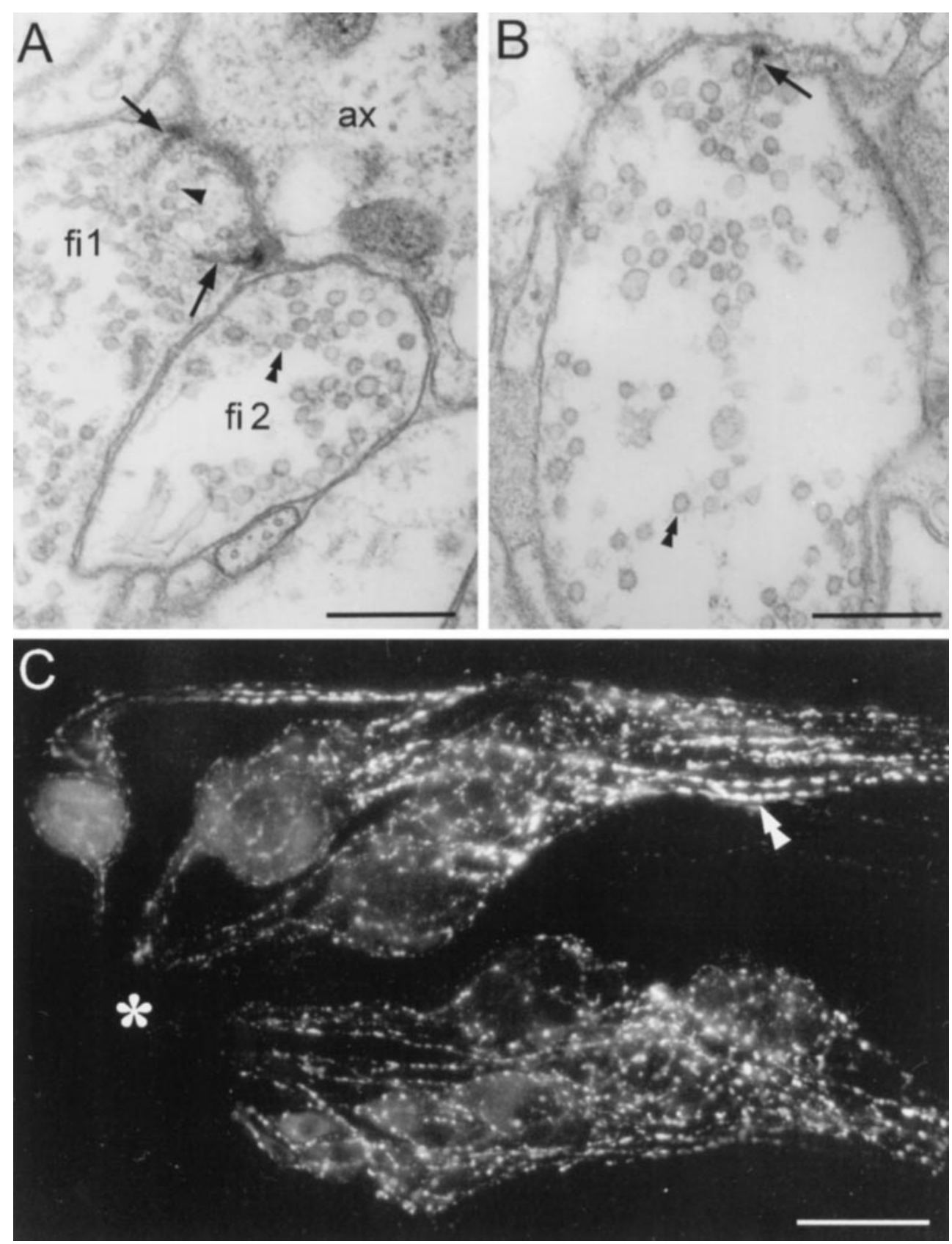

Figure 2. The distribution of peripheral synapses. $A$, Two types of synaptic contacts on mechanosensory neurons. Profile of a fine fiber ( $f i 1)$, with small, round electron-lucent synaptic vesicles $(\sim 26 \mathrm{~nm}$ diameter, type 1) (arrowhead) and two presynaptic dense bodies (arrows) opposite two postsynaptic elements, one in the axon region $(a x)$ of a mechanoreceptor in dyadic configuration. The second fiber element $\left(f_{i} 2\right)$ at one dyad is a profile with larger, round electron-lucent synaptic vesicles ( $\sim 2 \mathrm{~nm}$ diameter, type 2$)$ (double arrowhead). Magnification, 63,300×. Scale bar, $0.3 \mu \mathrm{m}$. B, Presynaptic site of a fiber profile with large synaptic vesicles (double arrowhead), resembling profile ( fi 2) in $A$ but with a conspicuous dense body (arrow). Magnification and scale bar as in $A$. $C$, Neuron pairs of lyriform organ VS-3, orientated as in Figure $1 C$, labeled with antibody against synapsin. Sites of presumed presynaptic specialization (double arrowhead) distribute along the length of all mechanosensory neurons. Asterisk, Dendrite tip region of the neurons. Magnification, $360 \times$. Scale bar, $50 \mu \mathrm{m}$. tical areas of the neurons. Only areas with clearly visible immunoreactive sites were used.

\section{RESULTS}

\section{Sensory neurons in VS-3 exhibit numerous peripheral synapses}

Our study concentrates on the mechanosensory neurons in the compound slit sense organ VS-3, which is located on the anterior side of the patella of all eight spider legs (Fig. $1 A$ ). The neurons in this organ have several important advantages for our investigations. The organ typically consists of seven to nine cuticular slits, which vary between 10 and $100 \mu \mathrm{m}$ in length (Fig. $1 B$ ). Each slit is associated with two bipolar mechanosensory neurons forming a neuron pair. The cell bodies of these neurons are up to 100 $\mu \mathrm{m}$ long and are thus exceptionally large (Fig. $1 C$ ). The receptors within each pair differ electrophysiologically, one being phasic and the other tonic (Seyfarth and French, 1994), so that the neurons can be identified individually. Dendrites, somata, and initial axon segments of the two neurons in each pair are enwrapped by a common glial sheath (Fig. $1 C$ ). They are embedded in the hypodermis, close to the cuticular slits in the periphery, and can easily be prepared as whole-mount preparations, as illustrated in Figure 1, $C$ and $D$. All sensory neurons in organ VS-3 receive numerous peripheral chemical synapses. Figure 2, $A$ and $B$, reveals that there are at least two types of chemical synapses, as characterized by their synaptic vesicle populations. The first type (type 1 synapses) contains primarily small, round electron-lucent vesicles (Fig. 2A), whereas the second type (type 2 synapses) contains large, round electron-lucent vesicles (Fig. $2 B$ ). Immunolabeling of sensory hypodermis preparations with a monoclonal antibody against the synaptic vesicle protein synapsin reveals 
linearly arranged punctate SYN-LIR across each mechanosensory neuron, as illustrated in Figure $2 C$. The specificity of antibody binding has been described previously (Fabian-Fine et al., 1999), suggesting that the IR-puncta represent the distribution of presynaptic sites across the sensory neurons. Figure $2 C$ demonstrates that most SYN-LIR puncta were located at the dendrites, cell bodies, and initial axon segments of the mechanosensory neurons, being most dense in the latter zone. The more proximal regions of the axons almost completely lack punctate immunoreactivity (Fabian-Fine et al., 1999). As shown further below, using the same antibody, immunostaining of neurons in trichobothria, tactile hairs, and internal joint receptors revealed a similar pattern of SYN-LIR along the three main segments (dendrites, somata, and initial axon regions) of all mechanosensory neurons.

\section{Histochemical identification of individual sensory neurons in organ VS-3}

The sensory neurons in each pair showed different histochemical staining intensities for the AChE-reaction product (Fabian and Seyfarth, 1997). Although one neuron was always stained faintly after AChE histochemistry, the electron-dense precipitate of enzyme activity in its partner neuron was much stronger. To distinguish the two electrophysiological types of neurons in each slit of organ VS-3, we used these differences to identify the neurons without the need to record from them electrophysiologically. This was essential for examining the detailed ultrastructural distribution of peripheral synapses on identified neurons, which required intact neurons and good tissue preservation. To correlate the intensity of $\mathrm{AChE}$ precipitate, faint or strong, in a neuron, with its electrophysiological type (slowly or rapidly adapting) (Fig. $3 A, B$ ), we first recorded from the neuron. Only those neurons were chosen that we could identify from a particular anatomical feature, such as its position or shape. Neurons were subsequently stained for AChE activity, as shown in Figure 3, C and $D$. The specificity of the AChE activity was confirmed previously from various control preparations (Fabian and Seyfarth, 1997).

All cells associated with the various slits of VS-3 that we have studied $(n=28)$ showed a perfect correlation between the neuron type and its AChE-staining intensity; the more intensely stained neuron was invariably the Type a neuron, whereas the Type b neuron was only faintly stained. Detailed investigations were made on the neuron pair associated with the second slit of VS-3. In this cell pair, the soma of one neuron is always very large (large neuron) and therefore easily distinguishable from the other neuron, which has a clearly smaller cell body (small neuron) (Fig. $1 C)$. Our investigations showed that in all preparations $(n=4)$ the small neuron was the rapidly adapting Type a neuron (Fig. $3 A$ ). In all AChE-stained preparations, the latter was the more intensely stained neuron (Fig. $3 C$ ). The more faintly stained large neuron (Fig. 3D) was always the slowly adapting Type b neuron. Intracellular recordings, without subsequent AChE staining, from such neurons in the second slit $(n>14)$ confirmed that the large neuron was always the Type $b$ neuron. Thus, we were able to identify reliably the electrophysiological type of all neurons on the basis of their AChE staining. As illustrated in Figure 4, satisfactory ultrastructural preservation was provided by the AChE-staining method; clearly visible differences in cytoplasmic staining between the two neurons were distinguished (Fig. 4A). Furthermore, different types of synapses identified previously in conventional EM sections (Fig. $2 A, B$ ) could be detected (Fig. 4B).
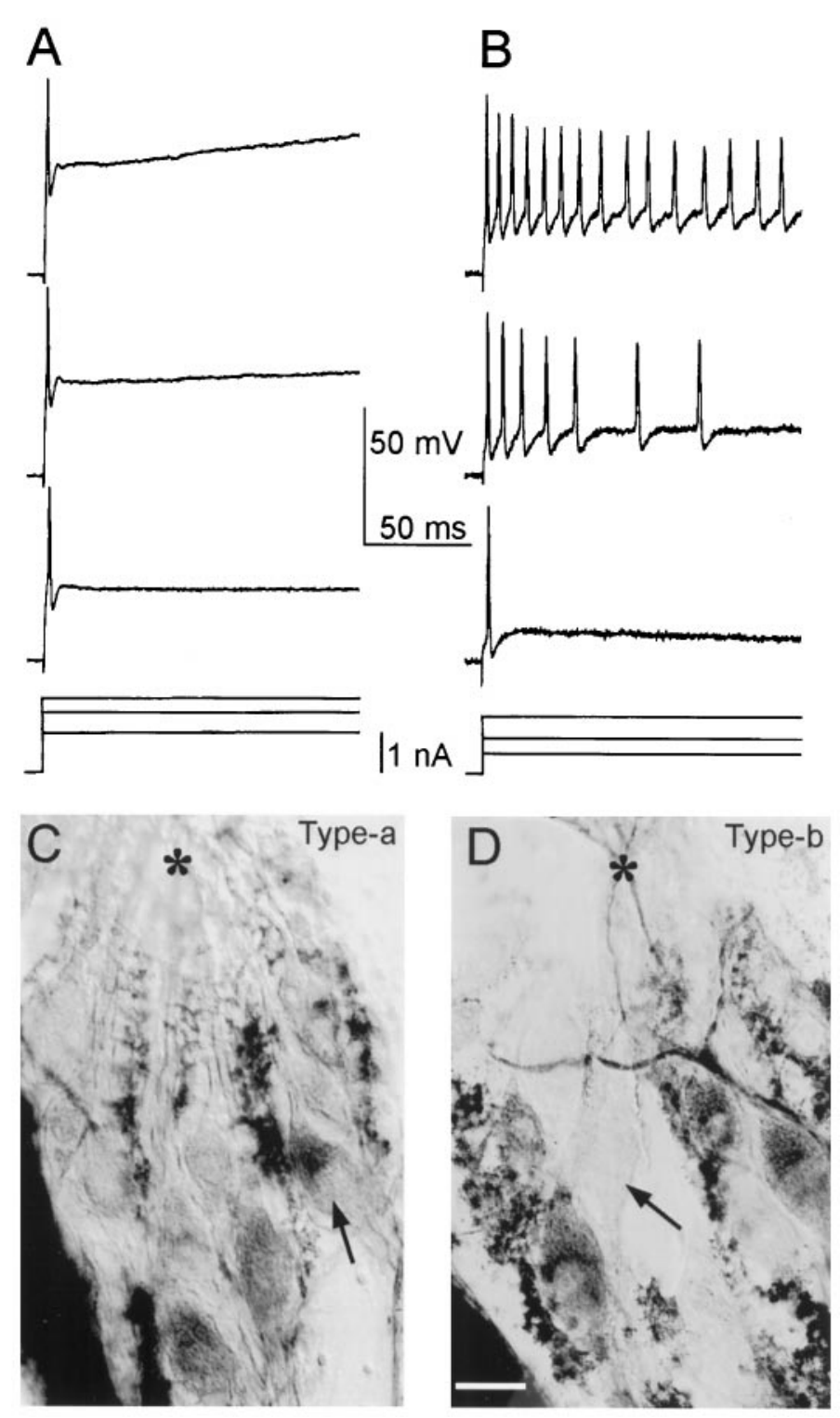

Figure 3. Correlation between the time course of electrophysiological adaptation $(A, B)$ and the intensity of AChE activity $(C, D)$ in VS-3 neurons. $A$, Rapidly adapting responses of Type a neuron after application of depolarizing current steps (bottom traces). Characteristically, this neuron generates only a single action potential, even at strong sustained depolarizations (threshold current to elicit spike, $\sim 1 \mathrm{nA}$; resting membrane potential, $-75 \mathrm{mV}$ ). $B$, Responses of a slowly adapting Type $b$ neuron, which produces characteristic bursts of action potentials (threshold current, $\sim 0.5 \mathrm{nA}$; resting membrane potential, $-66 \mathrm{mV}$ ). $C$, Intense AChE activity in the Type a neuron associated with slit 6 (arrow), which was recorded from in $A$. $D$, Faint AChE activity in the Type b neuron associated with slit 5 (arrow) recorded from in B. Asterisk, Dendritic region of mechanoreceptors. Magnification, $150 \times$. Scale bar: $C, D, 50 \mu \mathrm{m}$.

\section{The detailed distribution of peripheral synapses on identified mechanosensory neurons in VS-3}

To study the detailed distribution of the peripheral synaptic contacts on identified mechanosensory neurons, we reconstructed $10 \mu \mathrm{m}$ segments of EM series from each of three zones, the dendrite, the soma, and the initial axon segment, belonging to a neuron pair associated with the third slit in organ VS-3. Semithin $1 \mu \mathrm{m}$ sections were taken to reconstruct the areas in front of and behind the ultrathin sections. Thus, we were able to determine the 

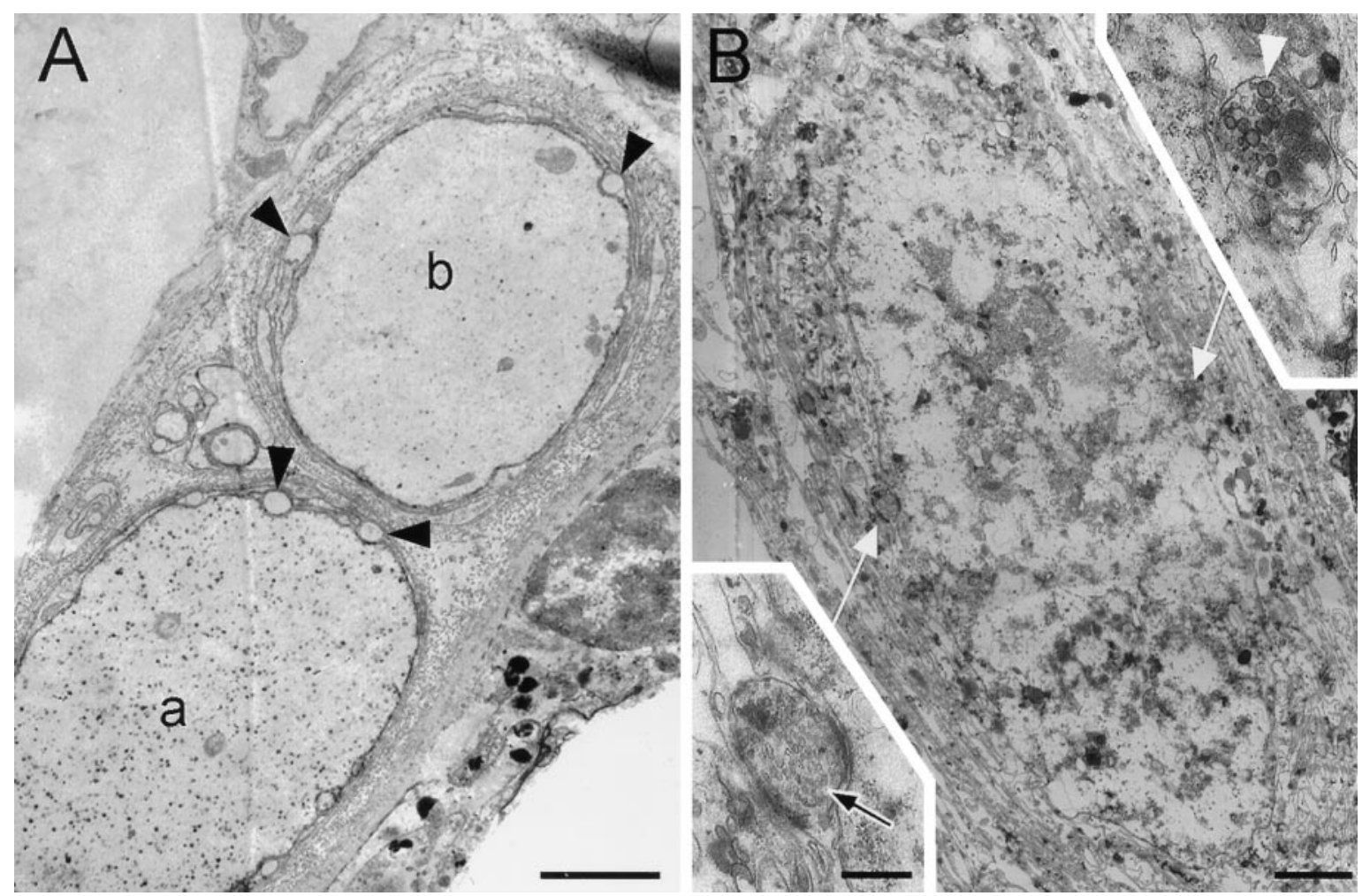

Figure 4. A, Electron microscopic cross section through the axon region of the two neurons in a VS-3 pair, revealing the darker AChE staining of a Type a neuron $(a)$ compared with its partner $(b)$. Profiles of fine fibers (arrowheads) surround both axons. Magnification, 7000×. Scale bar, $2 \mu \mathrm{m}$. $B$, The Type a neuron reconstructed in Figure 5, showing the two different types of synaptic sites that were identified by vesicle size; small (type 1, black arrow) or large (type 2, arrowhead) round vesicles, enlarged in the corresponding insets. Magnification, $4500 \times$. Scale bar, $2 \mu \mathrm{m}$. Inset, Magnification, 16,000 $\times$. Scale bar, $0.5 \mu \mathrm{m}$.

level of the $10-\mu \mathrm{m}$-ultrathin stacks relative to the cell bodies fairly precisely, as illustrated in Figure 5. The neurons were identified from their differential staining for AChE. Because the cell body of one neuron in each pair was always located distal to the cell body of its partner neuron (Fig. 1C), the relative locations of the sampled $10 \mu \mathrm{m}$ segments along the length of the reconstructed Type a neuron differed from those of the Type b neuron. In the Type a neuron (Fig. 5, red), the reconstructed areas were the basal region of the dendrite (Fig. $5 A$ ) and two different regions of the axon (Fig. $5 E, F$ ). In the Type b neuron (Fig. 5, purple), the reconstructed areas were the mid-dendritic region (Fig. 5D), the proximal region of the soma (Fig. $5 B$ ), and the initial axon segment region (Fig. 5C).

Both type 1 and type 2 presynaptic sites were frequently formed on each mechanosensory neuron in the reconstructed pair. The density of presynaptic sites over the neurons was approximately one per $0.5 \mu \mathrm{m}^{2}$ for the initial axon segment and less than a third of this value elsewhere. All observed peripheral synapses originated with presynaptic contacts from the small diameter fibers of unidentified origin, illustrated in Figures 2, $A$ and $B$, and $4 A$. These fibers primarily ran parallel to the longitudinal axis of the sensory neurons. In the following, we refer to them as "fine fibers." Fine fibers forming different synapse types were sometimes located close to each other (Fig. 2A), making contact to both the mechanosensory neuron and one another. Not only were unidirectional synapses observed, but some presynaptic fine fibers also formed reciprocal synaptic contacts onto each other. We also observed contacts from fine fibers that formed type 1 or type 2 synapses onto fine fibers that in turn formed synapses, either type 1 or type 2, onto the mechanosensory neurons. These established the following serial synapses: type 1 $\rightarrow$ type $2 \rightarrow$ mechanoreceptor; type $1 \rightarrow$ type $1 \rightarrow$ mechanoreceptor; and type $2 \rightarrow$ type $1 \rightarrow$ mechanoreceptor. Examples of a type $2 \rightarrow$ type $2 \rightarrow$ mechanoreceptor connection were not seen, although the profiles of two fibers with large vesicles were seen next to each other but without a visible active zone. Moreover, we found no evidence for lateral synaptic contacts directly between mechanosensory neurons (see Discussion).

In the axon and dendrite region, one particular fine fiber predominantly formed a string of large varicosities onto the mechanosensory neurons, with segments of narrow neurite connecting between consecutive varicosities (Fig. $5 C, D, F$ ). The small diameter of the neurites made the fine fibers hard to reconstruct in their entirety at the magnification adopted to reconstruct the mechanosensory neurons. We also could not readily determine the number of fine fibers from their neurites, partly because of the extremely small diameter of the neurites, but also because they were embedded in a dense glial sheath within which only their varicosities were conspicuous. To derive an estimate, we counted the numbers of the profiles of the fibers at higher magnification. There were six clear fiber profiles over the proximal axon, 13 over the initial axon segment, $\sim 20$ over the soma, and 12 along the dendrite. These were the minimum numbers of fiber profiles seen, and although we do not yet know the number of cells from which they derive, the fact that they increased going apically indicated that some fibers must branch. It is unclear whether an individual fiber forms synaptic contacts of only one type. The varicosities were of different sizes, ranging 


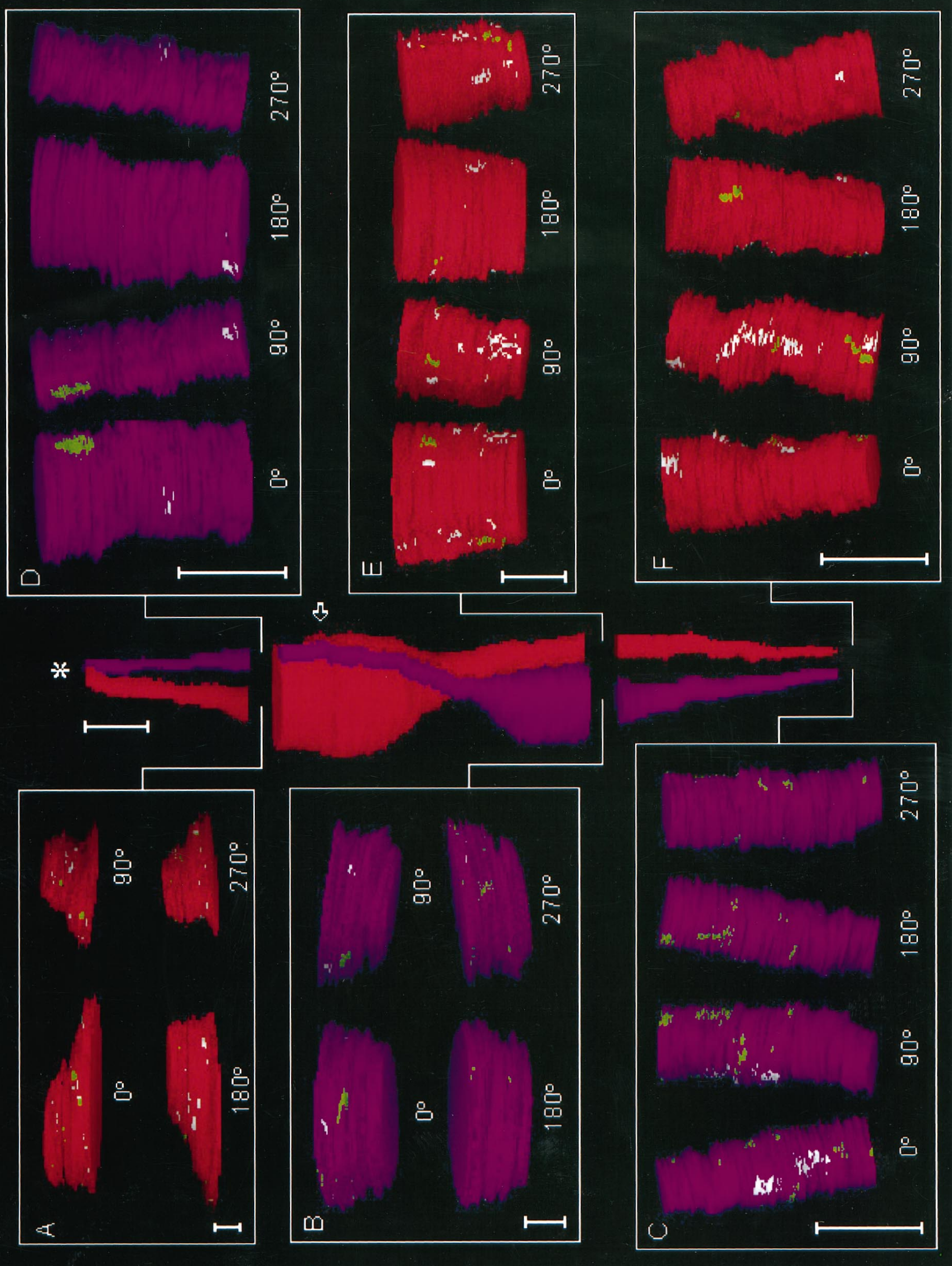




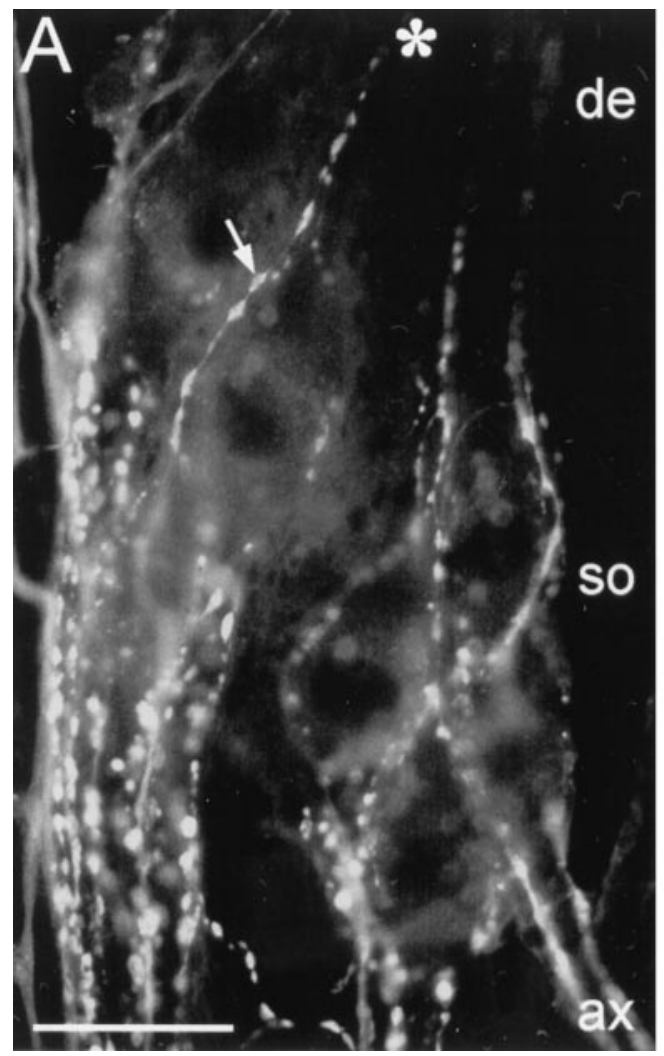

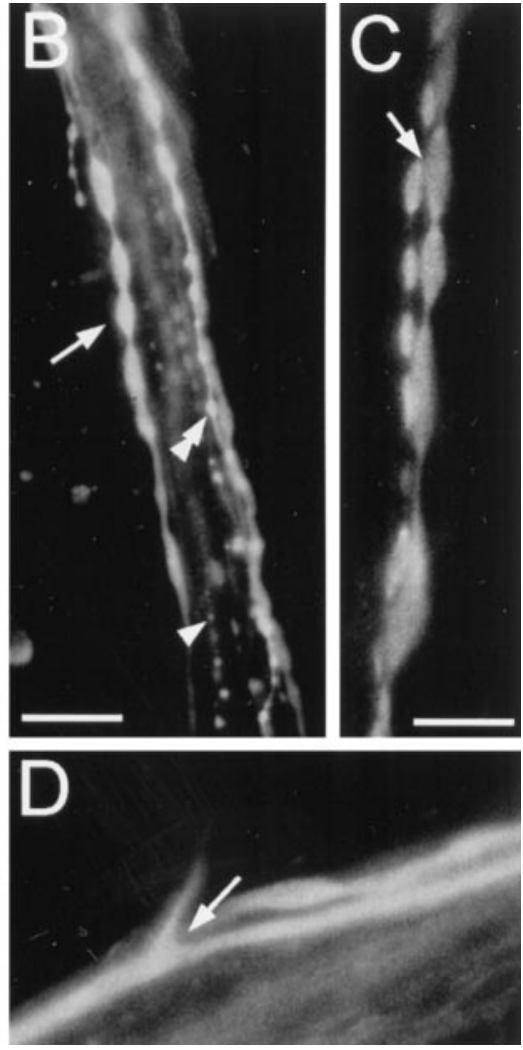

Figure 6. GABA-LIR fibers and varicosities extending across the neurons in organ VS-3; wholemount preparations labeled with Cy3. A, Hypodermis preparation demonstrating the density and distribution of fine GABA-LIR fibers. The fibers form rows of varicosities (arrow) at the axonal (ax), somatic (so), and dendritic (de) regions of the mechanosensory neurons. Asterisk, Dendrite tips extending toward the slit region. Magnification, $400 \times$. Scale bar, $50 \mu \mathrm{m}$. $B$, Initial axon segment region, illustrating three types of immunoreactive fibers and varicosities: thick (arrow), medium (double arrowhead), and thin (arrowhead). Magnification, $500 \times$. Scale bar, $20 \mu \mathrm{m}$. $C$, Higher magnification of two GABA-LIR fibers (coarse and medium) forming serial varicosities (arrow) in close proximity to each other. Magnification, $1000 \times$. Scale bar, $10 \mu \mathrm{m}$. $D$, Branching GABALIR fiber (arrow) at the axonal region of VS-3 neurons. Magnification and scale bar as in $C$. between 0.9 and $4.7 \mu \mathrm{m}$ in length on the particular neuron segments reconstructed in Figure $5 A-F$. These observations match the pattern of SYN-LIR and GABA-LIR seen in light microscopy (Figs. 2C, 6).

The synaptic contacts were distributed differentially across the surface of the mechanosensory neuron, in both longitudinal and radial directions. Over the soma region, the synaptic contacts seemed to be more evenly distributed than across the surfaces of both the dendrite and the initial segment of the axon where they were concentrated at relatively few sites. The number of synaptic inputs on the sensory neurons was greatest at the axon region, with fewer over the soma and least at the dendrite region. Around the circumference of the mechanosensory neurons, synapses on the axonal or dendritic regions were primarily located at one side of the sensory neurons, with the other side receiving less synaptic input (Fig. 5A, $C, D, F$ ). Both type 1 and type 2 contacts were present on both Type a and Type b neurons, but we found no clear difference in the pattern of relative distribution of the two types of synapses either between the two cells or across the surfaces of either.

\section{GABA-like immunoreactivity}

Treating neurons in all four types of sensilla (i.e., slit sense organs, tactile hairs, trichobothria, and joint receptors) with a polyclonal antibody against GABA revealed several intensely stained GABA-LIR fibers (Figs. 6, 7). All corresponded to the category of fine fiber described above. As an example of the mechanoreceptors in organ VS-3, Figure $6 A$ shows the GABALIR fibers running parallel to the longitudinal axis of the sensory neurons. They formed rows of varicosities, with narrow neurites between consecutive varicosities on the dendrites, somata, and initial axon segments. Most varicosities were located at the latter zone. There were no obvious differences in the pattern of GABALIR fibers between the Type a and Type $b$ neurons.

On the basis of the size and shape of their varicosities, three significantly different types of GABA-LIR fibers were seen, as illustrated in Figure $6 B$. The first fiber type (thick fiber) had primarily large varicosities, with a mean length of $8.5 \mu \mathrm{m}$ and a width of $2.6 \mu \mathrm{m}$ (Table 1), and were spaced at intervals of 1.3-23 $\mu \mathrm{m}$. The varicosities of the second fiber type (medium fiber) were smaller, with a mean length of $5.0 \mu \mathrm{m}$ and a width of $1.7 \mu \mathrm{m}$, and were spaced at intervals of $0.8-3.5 \mu \mathrm{m}$. The varicosities seen in the third fiber type (thin fiber) were smallest. They had a mean length of $1.7 \mu \mathrm{m}$ and a width of $1.1 \mu \mathrm{m}$ and were spaced at intervals of $0.6-5.3 \mu \mathrm{m}$. Often, two or more GABA-LIR fibers of different types paralleled each other, as shown in Figure $6 A-C$. At higher magnifications (Fig. 6C), the varicosities of individual fibers appeared to contact each other. This matches previous

\footnotetext{
Figure 5. Three-dimensional reconstructions of the distribution of synapses onto a neuron pair associated with the third slit in organ VS-3. The small-scale image of the neurons (center), reconstructed from serial semithin sections, is shown as viewed from the direction of the cuticular surface. The Type b neuron (purple) crosses over the Type a neuron (red) between the dendrite tips (asterisk) and the initial axon segments. Gaps (10- $\mu \mathrm{m}-\mathrm{long}$ ) in this reconstruction represent segments of the neurons that were serially sectioned at $75 \mathrm{~nm}$ thickness. Large-scale reconstructions of the corresponding EM series are shown in the six insets. Within each inset, four views of the reconstruction are shown at consecutive $90^{\circ}$ rotations about the longitudinal axis. The distribution of postsynaptic sites at each mechanosensory neuron is shown across the surface of the reconstructions, at synapses containing either small (white) or large ( green) round vesicles. Both synapse types are more numerous in the axonal than in the dendritic regions. Arrow, Level of the section shown in Figure $1 D$. Scale bars: $A-F, 5 \mu \mathrm{m}$; center, $20 \mu \mathrm{m}$.
} 

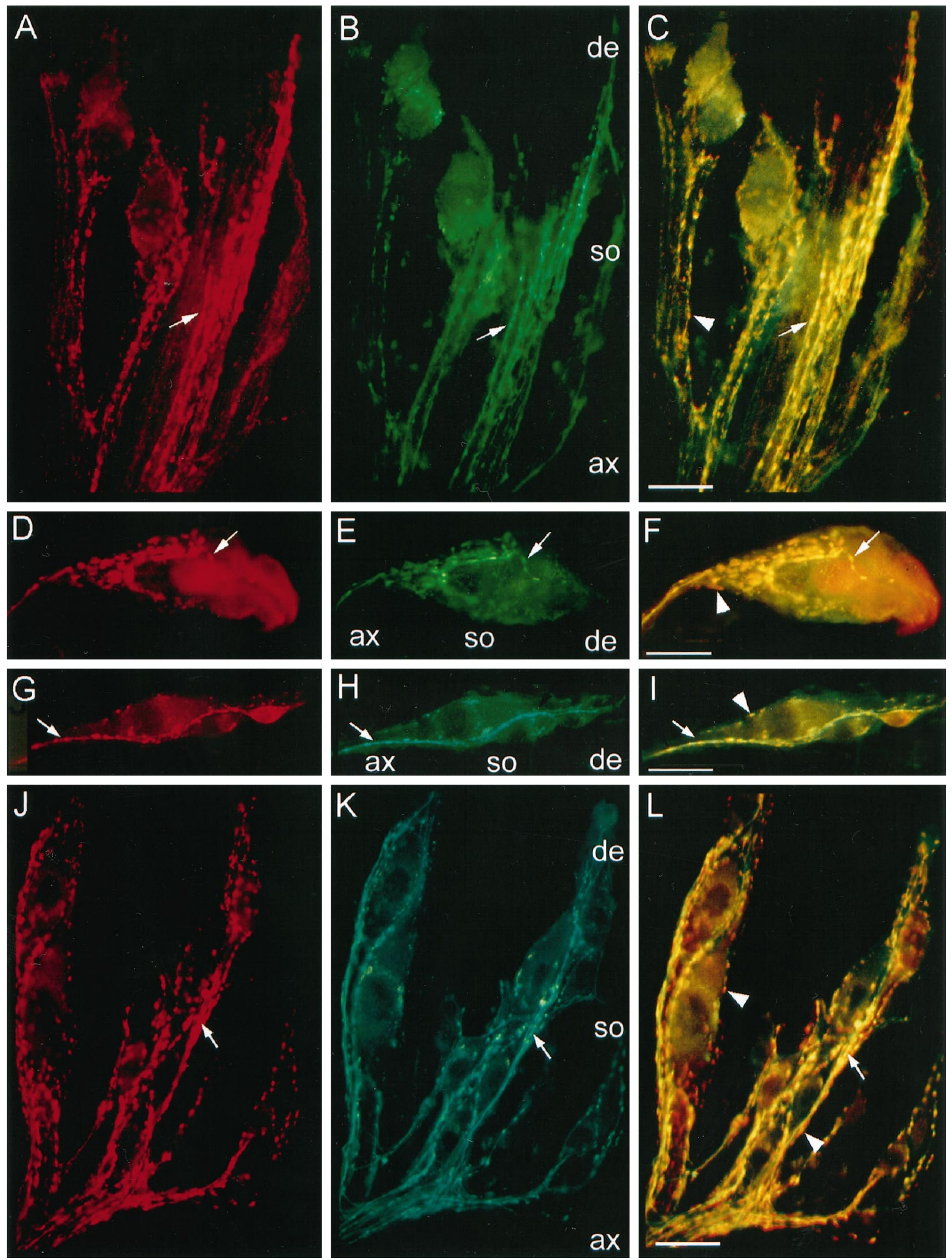

Figure 7. Immunofluorescent labeling of SYN-LIR and GABA-LIR in various mechanoreceptors of the spider leg. $A-C$, Lyriform organ VS-3; $D-F$, internal joint receptor; $G-I$, tactile hair; $J-L$, trichobothria. Red, Cy3-labeled SYN-LIR reveals fine fibers (arrows) extending across the neurons. Green, FITC-labeled GABA-LIR fibers (arrows). $C, F, I, L$, Double exposures of the same preparations. SYN-LIR and GABA-LIR are widely colocalized across the neurons (yellow), but numerous synapsin-LIR fibers show no overlapping GABA-LIR (arrowheads). ax, Initial axon segments; so, somatic regions; $d e$, dendritic regions. Magnification, $220 \times$. Scale bars, $50 \mu \mathrm{m}$. 


\begin{tabular}{|c|c|c|}
\hline & Length of varicosities $[\mu \mathrm{m}]$ & Width of varicosities $[\mu \mathrm{m}]$ \\
\hline Thick fiber & $8.5 \pm 2.5(4.7-14.5 ; n=29)$ & $2.6 \pm 0.65(1.8-3.9 ; n=29)$ \\
\hline Medium fiber & $5.0 \pm 1.2(2.7-7.3 ; n=27)$ & $1.7 \pm 0.6(0.7-3.3 ; n=27)$ \\
\hline Thin fiber & $1.7 \pm 0.5(0.7-2.5 ; n=25)$ & $1.1 \pm 0.5(0.4-2.1 ; n=25)$ \\
\hline
\end{tabular}

Mean values for length and width differ significantly in all three fiber types (one-sided $t$ test; $p<0.001$ in each case). Numbers in parentheses, range between extreme individual values and sample size, $n$.

ultrastructural observations showing serial synapses between varicosities (Fabian-Fine et al., 1999). Some of the GABA-LIR fibers branched in the initial axon segment region (Fig. 6D) and innervated more than one adjacent sensory neuron.

Control preparations (see Materials and Methods) were devoid of immunofluorescence, confirming the specificity of antibody binding. In addition, GABA-LIR neurons were seen in the ventral subesophageal ganglion of the CNS in aggregations typical of those described previously to be GABA-LIR (Schmid et al., 1990; Duncker, 1992). This strengthens the conclusion that the GABA antibody used here did indeed label authentic GABA and that the fine fibers of the mechanoreceptors did in fact contain GABA.

\section{GABA-synapsin double immunolabeling}

Given the similarities between SYN-LIR and GABA-LIR staining patterns, we investigated the possibility that these two epitopes were colocalized in the fine fibers. We performed GABA-synapsin double staining on neurons in slit sensilla, tactile hairs, trichobothria, and internal joint receptors. Figure 7 demonstrates that in all of the investigated receptor cells, SYNLIR and GABA-LIR were widely colocalized at all three main segments of the neurons (dendrite, cell body, and initial axon segment). All preparations displayed more SYN-LIR sites than GABA-LIR sites (Fig. 7), however, suggesting that not all SYNLIR sites also exhibited GABA-LIR. The approximate extent of colocalization between SYN-LIR and GABA-LIR in neurons of tactile hairs and trichobothria (see Materials and Methods) was counted at five different sample areas from neurons in three trichobothria and one tactile hair. Between 46 and $66 \%$ of all SYN-LIR sites colocalized with GABA-LIR sites. Thus, between 34 and $54 \%$ of the fine fibers forming peripheral synapses onto the sensory neurons were not GABA-LIR. There were also differences between the staining pattern of GABA-LIR and SYN-LIR sites. GABA-LIR labeled the cytoplasm and thus the entire fiber, whereas SYN-LIR stained only the varicosities, presumably at their presynaptic sites, and as a result appeared more punctate. Neurites between varicosities were not stained.

\section{The centrifugal origin of peripheral innervation}

To ascertain the origin of the peripheral innervation of the mechanosensory neurons, in particular to investigate whether the fine fibers derived from cell bodies in the CNS, we examined the effects of nerve section close to the CNS, at a site far from the somata of VS-3 mechanosensory neurons. This procedure allowed us to discriminate the rapid (orthograde) degeneration of the fine fibers, which we presumed to be efferent, from the much slower retrograde degeneration of the mechanosensory neurons.

Under the conditions used in our experiments in which the whole leg was amputated, retrograde degeneration of the afferent nerve fibers did not begin until 10-12 hr after lesion. In contrast, some profiles of the fine fibers exhibited signs of degeneration as early as $4 \mathrm{hr}$ after nerve section. Typical degeneration profiles after $8 \mathrm{hr}$ were electron-dense and filled with large vesicles, as shown in Figure $8 A$. The dendrites of the mechanosensory neurons were, in comparison, little changed from their appearance in control preparations (Fig. 8, de). Thus, degeneration occurred more rapidly in the fine fibers. The location of the nerve section during amputation was such as to cut the afferent axons of the mechanosensory neurons $\sim 12 \mathrm{~mm}$ from their subcuticular somata, at a place $\sim 4 \mathrm{~mm}$ from the CNS. Hence, the faster rate of degeneration among the fine fibers was compatible with the location of their somata in the CNS, indicating the centrifugal origin of this innervation. Interestingly, not all fine fibers showed fast degenerative changes. At intervals earlier than $10 \mathrm{hr}$ after nerve section, several fine profiles showed no apparent signs of degenerative change, indicating that such degeneration was not synchronous within all fine fibers (see Discussion).

\section{DISCUSSION}

There are six chief findings in this study. (1) The electrophysiological properties of slowly and rapidly adapting neurons in organ VS-3 correlate with their staining intensity for AChE. The electrophysiological type of each neuron can therefore be identified histochemically, without the need for intracellular recording. (2) All sensory neurons, in slit sensilla, tactile hairs, trichobothria, and internal joint receptors, are supplied by several GABA-LIR fibers of three different morphological types. These fibers form numerous varicosities at the dendritic, somatic, and initial axon regions of the sensory neurons, most densely over the latter zone. (3) The neurons in all four sensillum types investigated reveal punctate SYN-LIR. The distribution of such SYN-LIR is similar to the distribution of GABA-LIR sites across these neurons. (4) Double labeling shows that GABA-LIR and SYN-LIR widely colocalize at all sensory neurons investigated here. Some SYNLIR puncta are not GABA-LIR, however, indicating that they use (an)other neurotransmitter(s). (5) Three-dimensional reconstruction of the two mechanosensory neurons in a slit of organ VS-3 shows that all synapses originate from fine fibers running in parallel to the sensory neurons. Postsynaptic elements are mechanosensory neurons, glial cells, and fine fibers themselves. At least two different types of synaptic terminals are distinguishable with different sizes of synaptic vesicles, suggesting they release different neurotransmitters. The distribution of GABA-LIR and SYN-LIR has a pattern similar to the fine presynaptic fibers seen in EM reconstruction. (6) Degeneration studies are consistent with the efferent origin of the fine fibers from GABA-LIR somata in the CNS.

\section{AChE histochemistry}

It is not clear why the mechanosensory neurons express AChE activity or why they do so differentially. The staining intensity for AChE is not a size or volume effect. As demonstrated previously (Fabian and Seyfarth, 1997), many of the two neurons in a pair 

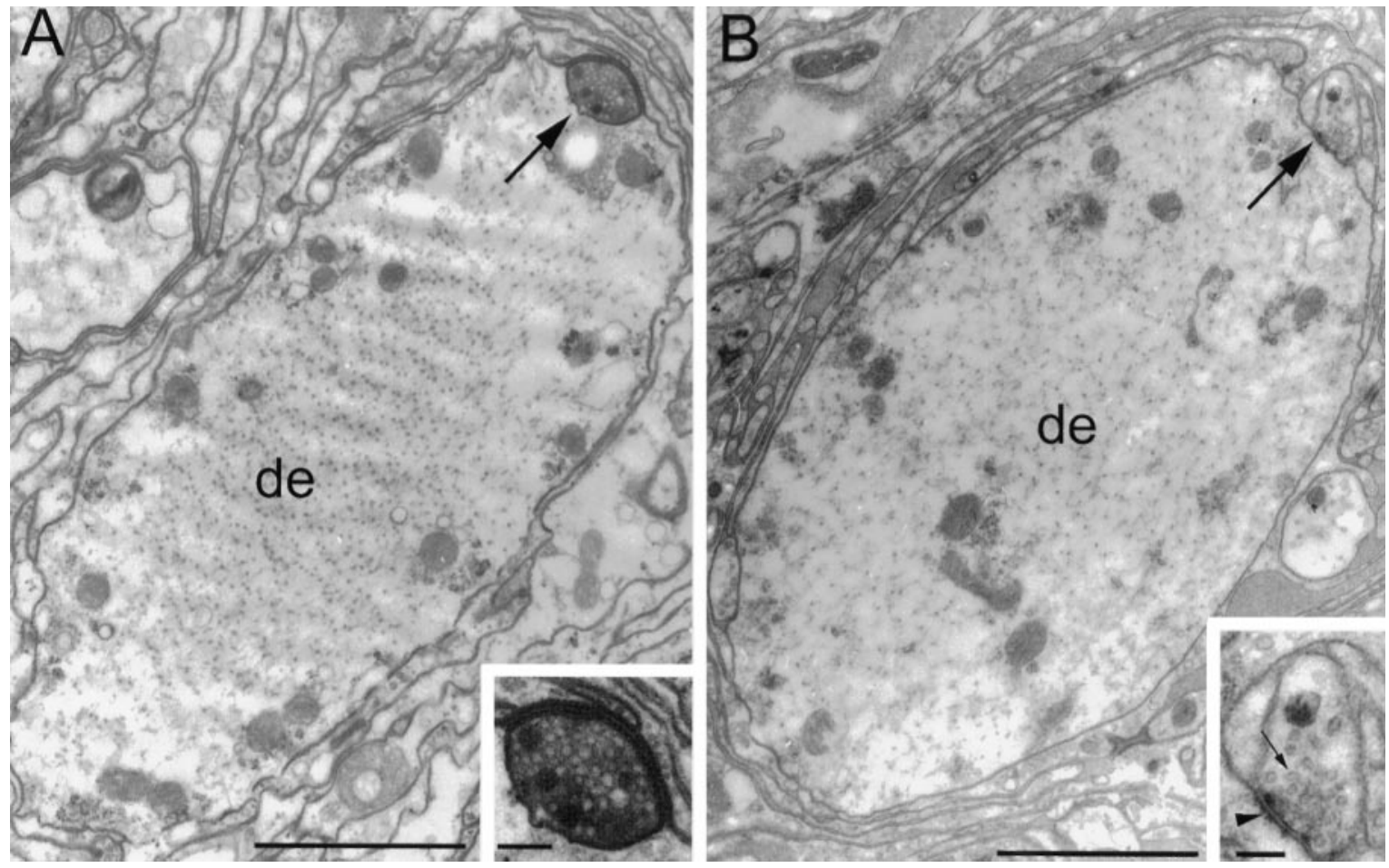

Figure 8. Degeneration of fine fiber profiles after nerve section. $A$, Dendrite region of a VS-3 neuron 8 hr after amputating the leg proximal to the sense organ. A fine fiber profile (arrow) abutting the mechanosensory neuron dendrite (de) is dark and contains numerous large degeneration vesicles. Magnification, 11,900×. Scale bar, $2 \mu \mathrm{m}$. Inset, Higher magnification of the degenerating profile. Magnification, 29,800×. Scale bar, $0.2 \mu \mathrm{m}$. $B$, The corresponding dendrite region in a control preparation that was fixed immediately via perfusion with EM fixative and not allowed to degenerate. A clear fiber profile containing electron-lucent synaptic vesicles (large arrow) forms a synaptic contact on the mechanosensory neuron dendrite (de). Magnification, 11,300×. Scale bar, $2 \mu \mathrm{m}$. Inset, Higher magnification of the fine fiber with its vesicles (small arrow) and the synaptic cleft (arrowhead). Magnification, 26,200×. Scale bar, $0.2 \mu \mathrm{m}$.

are of similar size but still show the clear difference in staining. Moreover, the darker neuron (Type a) is not always the smaller one of a pair. Only in the two neurons associated with slit 2, however, are the size, staining intensity, and mode of adaptation correlated consistently. Although the neurons may be cholinergic, insofar as they express choline acetyltransferase-like immunoreactivity (Fabian and Seyfarth, 1997) and their terminals may therefore have presynaptic cholinoceptors (Wonnacott, 1997), these should lie far away, in the CNS. It is possible that a large pool of AChE is stored differentially in one of the somata, but it may also be that the $\mathrm{AChE}$ reflects either nonsynaptic or even noncatalytic functions of AChE (Massoulié et al., 1993) not directly related to the particular rate of electrophysiological adaptation behavior of each neuron type.

\section{Immunocytochemistry}

There is good reason to suggest that punctate SYN-LIR at the neurons in all sensilla types investigated here represents the distribution of spider synapsin at presynaptic sites. The specificity of antibody binding has been established previously (Fabian-Fine et al., 1999). Furthermore, our present study shows that all sensory neurons are supplied by GABA-LIR fibers that form numerous varicosities onto the mechanoreceptors. The specificity of the GABA-antibody binding in spider tissue is confirmed by the absence of immunostaining in control preparations and by the presence of characteristically arranged GABA-LIR neurons in the subesophegeal ganglion. The fidelity of colocalization between the SYN-LIR and GABA-LIR patterns endorses the specificity of immunostaining in each. Furthermore, the staining pattern of both antibodies corresponds, in turn, with the general pattern of synapses reconstructed from serial EM. This suggests that some SYN-LIR sites at the neurons do indeed contain GABA and may therefore be GABAergic. Although there has been fine structural evidence for some time that sensory neurons in arachnids receive peripheral synaptic input (Foelix, 1975, 1985), this is the first demonstration of the distribution of GABALIR fibers.

\section{Efferent inhibitory pathways to mechanosensory neurons}

GABA mediates widespread inhibitory mechanisms in the nervous system (Roberts et al., 1976) and is widely distributed at the peripheral synapses of efferent innervations in various invertebrates. Examples include the neuromuscular junctions of nematodes (Johnson and Stretton, 1987), insects (Usherwood and Grundfest, 1965), and Crustacea (Kravitz et al., 1963). In addition, peripheral GABAergic innervation of sensory neurons in the crustacean muscle receptor organ (MRO) arises from efferent inhibitory innervation (Bazemore et al., 1957; Kuffler and Edwards, 1958; Elekes and Florey, 1987a), with a mechanism of action that is well investigated and particularly clear (Hagiwara et al., 1960). It does not seem unreasonable, therefore, to propose a similar inhibitory action for the fine GABA-LIR fibers reported here. Based on their ultrastructural investigations of internal joint receptors in spiders, Foelix and Choms (1979) have suggested that peripheral synapses may represent a pathway for central control that could inhibit receptor activity. From our degeneration tests, the origin of the presynaptic fine fibers appears to be 
central. There do remain, however, a few fine profiles that survive axotomy for longer than $8 \mathrm{hr}$. Examples are known in a number of other invertebrate nervous systems in which anucleate axons survive intact for long periods of time (for review, see Bittner, 1991). Although we cannot automatically assume the same phenomenon for such profiles in spiders, the only peripherally located neuron somata described are the mechanosensory neurons themselves, for which dye-fills (retrograde, as well as anterograde) show no evidence of axon collateral branches (Seyfarth et al., 1985). GABA immunostaining reveals numerous GABA-LIR perikarya in the subesophageal ganglion, but none in the periphery, and so confirms the efferent origin of these fibers. We therefore propose that the surviving small-fiber profiles seen after nerve section in our preparations are those of central neuron(s) in which degeneration is delayed.

\section{Distribution and differentiation of synaptic contacts}

The fine fibers at VS-3 neurons reconstructed from our EM series form linear rows of presynaptic varicosities, with consecutive presynaptic contacts that are concentrated on the initial axon segment. These findings correspond to the ultrastructural description of peripheral synapses at spider internal joint receptors provided previously by Foelix and Choms (1979). Inhibition at such proximal sites is widely seen in neurons (Shepherd, 1990) in which it is strategically located at the site of normal impulse initiation. So far, however, the region of lowest membrane threshold has not been investigated for spider mechanosensory neurons.

We observed three significantly different GABA-LIR fiber types on the basis of their varicosity sizes. This feature shares a similar pattern with the efferent innervation of sensory neurons of the crayfish MRO. After the initial description by Alexandrowicz (1951), Florey and Florey (1955) showed that the sensory neurons in this system are also supplied by three accessory fibers of different sizes; they are now known to be GABA-LIR (Elekes and Florey, 1987a).

Three features of the spider's peripheral innervation are especially significant for synaptic integration. The first is the size of the synaptic vesicles. The two sizes of synaptic vesicle (type 1 and type 2) (Figs. 2, 4) are presumed to contain different neurotransmitters. The smaller of two populations of round vesicles at the peripheral synapses on mechanoreceptors of the crustacean MRO (Nakajima and Reese, 1983) is known to contain GABA (Elekes and Florey, 1987a). We assume that the same is also true for the small vesicles of spider mechanoreceptors, whereas the contents of the large vesicles are not yet known. In the crustacean MRO, the second transmitter is glutamate (Takeuchi and Takeuchi, 1964), and it occurs at synapses with large round vesicles. It remains to be seen, however, whether glutamate is a second transmitter candidate at spider mechanoreceptors. The second feature at spider mechanoreceptors is that varicosities have widely differing sizes. Some varicosities are quite large, with numerous vesicles and multiple presynaptic dense bodies visible from EM series, whereas other varicosities contain few vesicles and have only a single dense body onto the sensory neurons. It seems likely that there are differences in transmitter output corresponding to the differences in the number of presynaptic dense bodies (Atwood and Cooper, 1995). Ultrastructurally, synapses with large synaptic vesicles are primarily small, containing relatively few synaptic vesicles. In comparison, synapses with small synaptic vesicles often extend over large distances, containing numerous vesicles. Differences in the size of the vesicle population presumably reflect differences in the sizes of the SYN-LIR puncta seen by light microscopy. The third feature is that numerous synaptic inputs are received not only by the mechanoreceptors but also form between the inputs themselves. Similar connections also occur between inputs at the stretch receptor neurons of the crayfish MRO (Hirosawa et al., 1981; Elekes and Florey, 1987b).

\section{Functional significance}

Assuming that the abundant peripheral synapses we describe include those that are GABAergic and inhibitory, a number of functional roles can be envisaged for the spider's mechanoreceptors. If such efferent fibers are activated simultaneously with motor fibers innervating nearby leg muscles, inhibition could serve to prevent spurious signals resulting from the spider's own movements, in much the same way, for instance, as for the neuromast organs of tailed vertebrates (Russell, 1971). Alternatively, a release from inhibition or excitation may increase the sensitivity of a receptor for its optimal stimulus, e.g., substrate vibration for the slit sensilla during courtship or prey localization (Barth, 1985). Given the number of efferent fibers, however, it seems likely that inhibitory interactions are more subtle than we can safely speculate from current evidence. An electrophysiological and pharmacological examination in single, identifiable mechanoreceptors (such as the VS-3 neurons) is now required to clarify the functional role of efferent innervation from GABA-LIR fibers.

\section{REFERENCES}

Alexandrowicz JS (1951) Muscle receptor organs in the abdomen of Homarus vulgaris and Palinurus vulgaris. Q J Microsc Sci 92:163-199.

Atwood HL, Cooper RL (1995) Functional and structural parallels in crustacean and Drosophila neuromuscular systems. Am Zool 35:556-565.

Barth FG (1985) Slit sensilla and the measurement of cuticular strains. In: Neurobiology of arachnids (Barth FG, ed), pp 162-188. Berlin: Springer.

Barth FG, Libera W (1970) Ein Atlas der Spaltsinnesorgane von Cupiennius salei Keys. Chelicerata (Araneae). Z Morphol Tiere 68:343-369.

Barth FG, Humphrey JAC, Wastl U, Halbritter J, Brittinger W (1995) Dynamics of arthropod filiform hairs. III. Flow patterns related to air movement detection in a spider (Cupiennius salei Keys.). Phil Trans $\mathrm{R}$ Soc Lond B Biol Sci 347:397-412.

Bazemore AW, Elliot KAC, Florey E (1957) Isolation of factor I. J Neurochem 1:334-339.

Bittner GD (1991) Long-term survival of anucleate axons and its implications for nerve regeneration. Trends Neurosci 14:188-193.

Duncker PM (1992) Vorkommen und Verteilung biogener amine im Zentralnervensystem der Jagdspinne Cupiennius salei Keyserling (Ctenidae, Aranae, Arachnida). Diplomarbeit, Formal- und Naturwissenschaftliche Fakultät. Wien, Austria: Universität Wien.

Eckweiler W, Seyfarth E-A (1988) Tactile hairs and the adjustment of body height in wandering spiders: behavior, leg reflexes, and afferent projections in the leg ganglia. J Comp Physiol [A] 162:611-621.

Elekes K, Florey E (1987a) Immunocytochemical evidence for the GABAergic innervation of the stretch receptor neurons in crayfish. Neuroscience 22:1111-1122.

Elekes K, Florey E (1987b) New types of synaptic connections in crayfish stretch receptor organs: an electron microscopic study. J Neurocytol 16:613-626.

Fabian F, Höger U, Seyfarth E-A, Meinertzhagen IA (1998) Threedimensional reconstruction of the peripheral synaptic connections of identified spider mechanosensory neurons. In: Göttingen neurobiology report (Proc 26th Göttingen Neurobiology Conference, Göttingen, Germany), Vol 2 (Elsner N, Wehner R, eds), p 585. Stuttgart, Germany: Verlag.

Fabian R, Seyfarth E-A (1997) Acetylcholine and histamine are transmitter candidates in identifiable mechanosensitive neurons of the spider Cupiennius salei: an immunocytochemical study. Cell Tissue Res 287:413-423.

Fabian-Fine R, Volknandt W, Seyfarth E-A (1999) Peripheral synapses 
at identifiable mechanosensory neurons in the spider Cupiennius salei: synapsin-like immunoreactivity. Cell Tissue Res, in press.

Fahrenbach WH (1985) Anatomical circuitry of lateral inhibition in the eye of the horseshoe crab, Limulus polyphemus. Proc R Soc Lond B Biol Sci 225:219-249.

Florey E, Florey E (1955) Microanatomy of the abdominal stretch receptors of the crayfish (Astacus fluviatilis, L.). J Gen Physiol 39:69-85.

Foelix RF (1975) Occurrence of synapses in peripheral sensory nerves of arachnids. Nature 254:146-148.

Foelix RF (1985) Sensory nerves and peripheral synapses. In: Neurobiology of arachnids (Barth FG, ed), pp 189-200. Berlin: Springer.

Foelix RF, Choms A (1979) Fine structure of a spider joint receptor and associated synapses. Eur J Cell Biol 19:149-159.

Foelix RF, Troyer D (1980) Giant neurons and associated synapses in the peripheral nervous system of whip spiders. J Neurocytol 9:517-535.

Hagiwara S, Kusano K, Saito S (1960) Membrane changes in crayfish stretch receptor neuron during synaptic inhibition and under action of gamma-aminobutyric acid. J Neurophysiol 23:505-515.

Harris DJ, Mill PJ (1977) Observations on the leg receptors of Ciniflo (Araneida: Dictynidae). I. External mechanoreceptors. J Comp Physiol 119:37-54.

Hartline HK, Ratliffe F (1972) Inhibitory interaction in the retina of Limulus. In: Handbook of sensory physiology, Vol V II/2, Physiology of photoreceptor organs (Fuortes MGF, ed), pp 381-447. Berlin: Springer.

Hirosawa K, Tao-Cheng J-H, Nakajima Y, Tisdale AD (1981) Thinsection and freeze-fracture studies of crayfish stretch receptor synapses including the reciprocal inhibitory synapse. J Comp Neurol 200:39-53.

Höger U, Seyfarth E-A (1995) Just in the nick of time: postembryonic development of tactile hairs and of tactile behavior in spiders. Zoology 99:49-57.

Humphrey CD, Pittman FE (1974) A simple methylene blue-azure IIbasic fuchsin stain for epoxy-embedded tissue sections. Stain Technol 49:9-14.

Johnson CD, Stretton AOW (1987) GABA-immunoreactivity in inhibitory motor neurons of the nematode Ascaris. J Neurosci 7:223-235.

Karnovsky MJ, Roots L (1964) A "direct-coloring" thiocholine method for cholinesterases. J Histochem Cytochem 12:219-221.

Klagges BRE, Heimbeck G, Godenschwege TA, Hofbauer A, Pflugfelder GO, Reifegerste R, Reisch D, Schaupp M, Buchner S, Buchner E (1996) Invertebrate synapsins: a single gene codes for several isoforms in Drosophila. J Neurosci 16:3154-3165.

Kravitz EA, Kuffler SW, Potter DD (1963) Gamma-aminobutyric acid and other blocking compounds in Crustacea. III. Their relative concentrations in separated motor and inhibitory axons. J Neurophysiol 26:739-751.

Kuffler SW, Edwards C (1958) Mechanisms of gamma aminobutyric acid (GABA) action and its relation to synaptic inhibition. J Neurophysiol 21:589-610.

Livingston RB (1959) Central control of receptors and sensory transmission systems. In: Handbook of physiology, Sect 1, Neurophysiology, Vol I (Magoun HW, ed), pp 741-760. Washington, DC: American Physiological Society.

Maier L, Root TM, Seyfarth E-A (1987) Heterogeneity of spider leg muscle: histochemistry and electrophysiology of identified fibers in the claw levator. J Comp Physiol [B] 157:285-294.

Massoulié J, Pezzementi L, Bon S, Krejci E, Vallette F-M (1993) Molecular and cellular biology of cholinesterases. Prog Neurobiol 41:31-91.
Meinertzhagen IA, Hu X (1996) Evidence for site selection during synaptogenesis: the surface distribution of synaptic sites in photoreceptor terminals of the flies Musca and Drosophila. Cell Mol Neurobiol 16:677-698.

Meinertzhagen IA, Govind CK, Stewart BA, Carter JM, Atwood HL (1998) Regulated spacing of synapses and presynaptic active zones at larval neuromuscular junctions in different genotypes of the flies Drosophila and Sarcophaga. J Comp Neurol 393:482-492.

Mulloney B, Hall WM (1990) GABA-ergic neurons in the crayfish nervous system: an immunocytochemical census of the segmental ganglia and somatogastric system. J Comp Neurol 291:383-394.

Nakajima Y, Reese TS (1983) Inhibitory and excitatory synapses in crayfish stretch receptor organs studied with direct rapid-freezing and freeze-substitution. J Comp Neurol 213:66-73.

Rathmayer W, Koopmann J (1970) Die Verteilung der Propriorezeptoren im Spinnenbein. Z Morphol Tiere 66:212-223.

Reissland A, Görner P (1985) Trichobothria. In: Neurobiology of arachnids (Barth FG, ed), pp 138-161. Berlin: Springer.

Roberts E, Chase TN, Tower DB (1976) GABA in nervous system function. New York: Raven.

Russell IJ (1971) The role of the lateral-line efferent system in Xenopus laevis. J Exp Biol 54:621-641.

Schmid A, Duncker M, Spörhase-Eichmann U (1990) Verteilung FMRFamid- und GABA-artiger Immunoreaktivität im ZNS der Jagdspinne Cupiennius salei. Verh Dtsch Zool Ges 83:639-640.

Seguela P, Geffard M, Buijs RM, Le Moal M (1984) Antibodies against $\gamma$-aminobutyric acid: specificity studies and immunocytochemical results. Proc Natl Acad Sci USA 81:3888-3892.

Seyfarth E-A (1985) Spider proprioception: receptors, reflexes, and control of locomotion. In: Neurobiology of arachnids (Barth FG, ed), pp 230-248. Berlin: Springer.

Seyfarth E-A, French AS (1994) Intracellular characterization of identified sensory cells in a new spider mechanoreceptor preparation. J Neurophysiol 71:1422-1427.

Seyfarth E-A, Eckweiler W, Hammer K (1985) Proprioceptors and sensory nerves in the legs of a spider, Cupiennius salei (Arachnida, Araneida). Zoomorphology 105:190-196.

Seyfarth E-A, Hammer K, Spörhase-Eichmann U, Hörner M, Vullings HGB (1993) Octopamine immunoreactive neurons in the fused central nervous system of spiders. Brain Res 611:197-206.

Shepherd GM, Koch C (1998) Introduction to synaptic circuits. In: The synaptic organization of the brain, Ed 4 (Shepherd GM, ed), pp 1-36. New York: Oxford UP.

Takeuchi A, Takeuchi N (1964) The effect on crayfish muscle of iontophoretically applied glutamate. J Physiol (Lond) 170:296-317.

Usherwood PNR, Grundfest H (1965) Peripheral inhibition in skeletal muscle of insects. J Neurophysiol 28:497-518.

Walrond JP, Govind CK, Huestis SE (1993) Two structural adaptations for regulating transmitter release at lobster neuromuscular synapses. J Neurosci 13:4831-4845.

Wickelgren WO, Leonard JP, Grimes MJ, Clark RD (1985) Ultrastructural correlates of transmitter release in presynaptic areas of lamprey reticulospinal axons. J Neurosci 5:1188-1201.

Wonnacott S (1997) Presynaptic nicotinic ACh receptors. Trends Neurosci 20:92-98.

Zill SN, Seyfarth E-A (1996) Exoskeletal sensors for walking. Sci Am 275:86-90. 